\title{
BMSC cells alleviate liver injury induced by ulcerative colitis via repairing the intestinal-liver barrier and activating hepatocyte growth factor
}

\section{Zhejun Deng}

Guangxi Medical University

\section{Qifang Zhang}

Nanxishan Hospital of Guangxi Zhuang Autonomous Region

\section{Yunchao Meng}

Nanxishan Hospital of Guangxi Zhuang Autonomous Region

Lei Zhao

Nanxishan Hospital of Guangxi Zhuang Autonomous Region

Wenjie Luo

Guilin Medical University

Jing Zhang

Nanxishan Hospital of Guangxi Zhuang Autonomous Region

Jie Hu

Guilin Medical University

Haixing Jiang ( $\nabla$ gx_jhxing@163.com )

Guangxi Medical University

\section{Research}

Keywords: Ulcerative colitis (UC), bone marrow-derived mesenchymal stem cells (BMSCs), liver injury.

Posted Date: February 23rd, 2021

DOI: https://doi.org/10.21203/rs.3.rs-210008/v1

License: (1) (1) This work is licensed under a Creative Commons Attribution 4.0 International License. Read Full License 


\section{BMSC cells alleviate liver injury induced by ulcerative colitis via repairing the intestinal-liver barrier and activating hepatocyte growth factor}

Zhejun Deng ${ }^{1+}$, Qifang Zhang ${ }^{2+}$, Yunchao Meng ${ }^{2}$, Lei Zhao ${ }^{2}$, Wenjie Luo ${ }^{3}$, Jing Zhang ${ }^{2}$, Jie $\mathrm{Hu}^{3}$, Haixing Jiang ${ }^{1^{*}}$

*Correspondence: gx jhxing@163.com

+Zhejun Deng and Qifang Zhang contributed equally to this work

${ }^{1}$ Guangxi Medical University, Nanning 530021, China.

Full list of author information is available at the end of the article

\section{Abstract}

Background: Ulcerative colitis (UC), belongs to inflammatory bowel diseases (IBD), frequently induces liver inflammation and injury. Previous studies have proved that bone marrow-derived mesenchymal stem cells (BMSCs) can suppress inflammation and improve intestinal mucosal injury in colitis, however, the effects of BMSCs on colitis-induced liver injury and the underlying molecular mechanisms remain unclear. Here, we investigated the effects and mechanisms of BMSCs in acute ulcerative colitis Balb/C mice, which were induced by $4 \%$ dextran sodium sulphate (DSS).

Methods: In this study, BMSCs derived from Balb/C mice were administrated by single intravenous injection with a dose of $5^{\star} 10^{\wedge} 7$ cells $/ \mathrm{kg}$. And then, the effects and underlying molecular mechanisms were investigated. Firstly, degree of liver injury in colitis mice was evaluated by hepatic ALT, AST, ALP and TBIL levels, which were measured by specific determination kits, the levels of TNF- $\alpha$, IL-6, IFNY and LPS were examined by ELISA. Secondly, as the indicator of intestinal-liver barrier disorder, tight junction proteins were analyzed by western blot. Thirdly, the pathological changes in colon and liver were detected by H\&E staining. At last, homing of BMSCs to lesion tissues was investigated by Immunofluorescence.

Results: The results indicated that histopathological changes in model mice had been greatly alleviated, BMSCs infusion remarkably decreased the serum ALT, AST, ALP and TBIL level, and meanwhile reduced pro-inflammatory cytokines in liver tissues. Furthermore, homing of BMSCs was observed in colon and liver, and the disorder of intestinal-liver barrier was declined significantly.

Conclusions: In conclusion, BMSCs alleviate liver injury induced by ulcerative colitis via repairing the intestinal-liver barrier and activating hepatocyte growth factor, it has potential application prospects in the treatment of liver injury induced by ulcerative colitis.

Keywords: Ulcerative colitis (UC), bone marrow-derived mesenchymal stem cells (BMSCs), liver injury. 


\section{Introduction}

Ulcerative colitis (UC) is an inflammatory bowel disease (IBD) restricted to the colonic mucosa and submucosa with characteristic ulcers or open sores [1]. UC is a commonly occurring disease, and has a prevalence of 7.6-246 per 100000 individuals in the worldwide [2]. The etiology of UC is still poorly understood, which is likely to involve immune dysfunction [3], genetic susceptibility [4] and microbial imbalance [5]. So far, due to the high recurrence rate, the clinical therapeutic efficacy of UC is still unsatisfactory [6].

Numerous of evidence indicates that the most typical character of UC is the abnormal immune response in the intestinal wall with epithelial barrier disruption [7-10]. Studies show that abnormal immune response of intestinal wall mucosa and intestinal mucosal barrier defection can cause liver injury by over exposure to bacterial translocation, enteric antigens and toxins, as well as inflammatory mediators [11-15]. As for the pathogenesis of liver injury induced by colitis, it is well known that enteric toxins are more convenient to enter the liver through the portal vein, leading to liver diseases [16]. More seriously, this pathogenic process is usually accompanied by the destruction of liver barrier function, which aggravates the risk of liver injury [17]. Regrettably, the drugs currently used to treat UC were reported have some unsatisfactory gastrointestinal and liver adverse effects [18-20]. Therefore, it is meaningful to find better treatments that can treat liver damage caused by UC.

In the recent years, systemic administration of BMSCs to regulate the immune response and enhance endogenous regeneration is becoming a new treatment strategy in immune hyperactivity and tissue injury diseases [21-23]. Intravascular injection of BMSCs has been confirmed to be an effective treatment for autoimmune diseases [24-25], graft vs. host disease (GVHD) [26-27], vascular diseases and diabetes [28-29]. Hundreds of clinical trials of BMSC cell therapy are now being carried out world-wide [30-32]. Meanwhile, studies have showed that BMSCs can homing to injured intestinal crypt and promote intestinal epithelial regeneration, as well as repair intestinal epithelial tight junction in DSS induced colitis mice [33]. In our previous studies, we have proved BMSCs could inhibit hepatic fibrosis and repair liver injury via activating hepatocyte growth factor (HGF). However, the role of BMSCs in colitis induced liver injury, as well as how BMSCs will work are still unknown. Undoubtedly, these problems are very urgent and will have great clinical significance. Based on this, in this study, we aimed to confirm the effects of BMSCs in colitis induced UC and the underlying mechanism. 


\section{Materials and methods}

\section{Reagents}

Dextran sodium sulfate (DSS; molecular weight: 36,000-50,000) was purchased from MP Biomedicals (Santa Ana, CA, USA). The aminotransferase (AST), alanine aminotransferase (ALT), alkaline phosphatase (ALP) and total bilirubin (TBIL) testing kits were provided by Jiancheng Institute of Biotechnology (Nanjing, China). Enzyme-linked immunosorbent assay (ELISA) kits of TNF-a, IL-6 and IFN $\gamma$ was obtained from Sigma-Aldrich (St. Louis, MO, USA). The ELISA kits for HGF and HGFA were respectively purchased form R\&D systems (Minneapolis, MN, USA) and Cloud-Clone Corp (Houston, TX, USA). The ELISA kits for LPS was obtained from Shanghai Enzyme-linked Biotechnology Co., Ltd (Shanghai, China). Primary antibodies specific for claudin1、claudin2、claudin5、claudin7、occludin、 ZO-1 were purchased from Abcam (Cambridge, UK), and Primary antibodies specific for HGF $\alpha$ chain was purchased from Santa Cruz Biotech (Santa Cruz, CA, USA).

\section{Isolation and culturing of BMSCs}

The BMSCs were isolated and cultured following the published protocol as mentioned [34]. In this present study, the bone marrow was obtained from the hind limbs of male Balb/C mice aged 5-6 weeks by flushing with Dulbecco's modified Eagle's medium (DMEM) medium supplemented with $10 \%$ fetal bovine serum (Hyclone, Salt Lake City, UT, USA), 100 units/ml penicillin and 100 units/ml streptomycin (Sigma-Aldrich, St-Louis, MO, USA). The cells were cultured in a humidified incubator at $37^{\circ} \mathrm{C}$ under an atmosphere with $5 \% \mathrm{CO} 2$ and the third generation of BMSCs were used in the experiments. FITC-marked rabbit anti-mouse CD29 or CD34 (BD, NJ, USA) and PE-linked anti-mouse CD45 or CD90 antibody (BD, NJ, USA) were used to stain cell surface markers which subsequently were evaluated using fluorescence-activated cell sorting with flow cytometry. Oil Red O was used to assess cell capability of adipogenesis. The capability of osteogenesis was evaluated using calcium tubercle sodium alizarinsulfonate staining.

\section{BMSCs labeling}

BMSCs were maintained in DMEM supplemented with $0.1 \mathrm{mmol} / \mathrm{L}$ 5-bromo-2'-deoxyuridine (BrdU, Sigma-Aldrich, St. Louis, MO, USA), 10\% fetal bovine serum (FBS, Gibco, Carlsbad, CA, USA), 100 units $/ \mathrm{ml}$ penicillin and 100 units $/ \mathrm{ml}$ streptomycin for 3 days, after which medium was changed to complete DMEM without BrdU. 
A total of 45 6-week-old male BALB/C mice were obtained from shanghai SLAC laboratory animal Co., Ltd. The animals were administered a standard rodent diet with free access to water (ad libitum) and were housed in rooms sustained at $22 \pm 1{ }^{\circ} \mathrm{C}$ with a $12 \mathrm{~h}$ light/dark cycle. The experimental protocol was reviewed and approved by the Animal Ethics Committee of Guilin Medical University (No. 20140304026). To evaluate the protective effects of BMSCs on liver injury induced by ulcerative colitis, mice were randomly divided into the three experimental groups ( $n=15$ per group): control group, model+PBS (4\% DSS) group, DSS+PBMCs $\left(5^{\star} 10^{\wedge} 7\right.$ cells $\left./ \mathrm{kg}\right)$ group. The control group was given distilled water every day, and mice in the other groups received DSS (molecular mass 36000-50000, MP Biomedicals) added at $4 \%$ concentration to the drinking water within 7 consecutive days. On the seventh day, mice in model+PBS group were injected with BrdU labeled BMSCs $\left(5^{\star} 10^{\wedge} 7\right.$ cells $\left./ \mathrm{kg}\right)$ via tail vein, and mice in model+PBS group were injected with same volume of PBS via tail vein. From the eighth day, all the mice in three groups were administrated with distilled water. Peripheral blood was selected on day 7, 9, 11, 15, 19, 23 for biochemical detection. The mice in each group were sacrificed in batches on day 9, 16, 23, colon and liver samples were collected, a portion of the tissue was fixed in $4 \%$ paraformaldehyde for histopathological analyses, and the remaining was stored at $-80^{\circ} \mathrm{C}$ for further immunofluorescence staining and immunoblot assays.

\section{Biochemical analyses}

Serum were separated from blood after centrifugation at $4000 \mathrm{rpm}$ for $5 \mathrm{~min}$ and stored at $-80{ }^{\circ} \mathrm{C}$ until use. The serum levels of alanine transaminase (ALT), aspartate aminotransferase (AST), alkaline phosphatase (ALP) and total bilirubin (TBIL) were measured with an enzyme-linked immunosorbent assay (ELISA) kit according to the manufacturer's instructions.

\section{Enzyme-linked immunosorbent assay (ELISA)}

The levels of TNF- $\alpha$, IL-6, IFN $\gamma$, HGF, HGFA in serum or liver tissues were analyzed by corresponding ELISA kits, according to their manufacturer's instructions. The optical density of each well was measured at $450 \mathrm{~nm}$.

\section{Histopathological Evaluation}

After being fixed in $10 \%$ formalin solution for $24 \mathrm{~h}$, the colon and liver tissues were embedded in paraffin and sectioned $(5 \mu \mathrm{m})$ with a rotary microtome. For histological assessment of tissue damage, formalin-fixed, paraffin-embedded tissue sections were deparaffinized with xylene and stained with hematoxylin and eosin (H\&E). Stained slides were evaluated under a light microscope by two 
independent medical technologists.

\section{Immunofluorescence staining}

For immunofluorescence staining, prepared frozen slices were fixed with iced acetone for $10 \mathrm{~min}$ and permeabilized with $0.3 \%$ Triton-X for $30 \mathrm{~min}$ at room temperature, the tissue sections were then incubated overnight at $4^{\circ} \mathrm{C}$ with rat anti-BrdU (abcam, Cambridge, UK) antibodies. Subsequently, the cells were incubated with Alexa 488-conjugated secondary antibody (Invitrogen, Carlsbad, CA, USA) at

RT. The slides were counterstained with DAPI. The images were observed with a fluorescence microscope EVOS (Thermo Fisher Scientific, CA, USA). All experiments were performed in triplicate.

\section{Western blotting}

Proteins were extracted from the colon and liver tissue samples by RIPA reagent containing 1mM PMSF and phosphatase inhibitors, and the concentrations were detected by BCA protein assay kit. Equal amounts of protein were separated on $10 \%$ SDS-PAGE electrophoretic gels and transferred to PVDF membrane. Subsequently, the membranes were blocked with $5 \%$ BSA at room temperature for $2 \mathrm{~h}$ and incubated overnight at $4{ }^{\circ} \mathrm{C}$ with a $1: 1000$ dilution of different primary antibodies. The membranes were then washed three times with TBST followed by incubation with appropriate HRP-conjugated secondary antibodies for $1 \mathrm{~h}$ at room temperature. The membranes were visualized with an enhanced chemiluminescence kit.

\section{Statistical analysis}

Results are presented as means \pm standard deviations (SD) of three independent experiments. Significant differences in the mean values were evaluated by unpaired t-test. One-way ANOVA was used to compare continuous variables among two or more groups. $p<0.05$ was considered statistically significant.

\section{Results}

The intestinal-liver barrier was disrupted in ulcerative colitis induced liver injury model

In the present study, we applied $4 \%$ DSS to induce mice colitis model, which has been reported to cause adverse liver reactions. Changes in disease characteristics such as body weight, colon length, as well as pathological changes in colon all indicated that ulcerative colitis model was successfully constructed (Fig 1a-c). Consistent with previous research reports, histopathological changes in liver tissue were frequently observed in ulcerative colitis mice (Fig 1c). Meanwhile, the serum ALT, AST, TC levels were significantly 
increased in model mice (Fig 1d-g), which also revealed that we successfully established ulcerative colitis caused liver injury model.

To determine the intestinal-liver barrier dysfunction in ulcerative colitis induced liver injury model, we detected intestinal-liver barrier related proteins in colon and liver respectively. As showed in Fig 2, claudin2、 claudin 5、 claudin 7, as well as occludin and ZO-1 were remarkably decreased in colon and liver, while the expression of claudin1 was down-regulated in liver only. These results well confirmed ulcerative colitis induced intestinal-liver barrier dysfunction.

BMSCs alleviated the syndrome of ulcerative colitis and improved histopathologic indices.

To assess the effects of BMSCs, we investigated the typical symptoms of ulcerative colitis. After treatment with BMSCs, the body loss of model mice was markedly reversed from Day 9 (Fig 3a). Meanwhile, compared with Model+PBS group, BMSCs extremely increased the colon length (Fig 3b). As showed in Fig 3c-d, BMSCs treatment significantly improved inflammatory infiltration, and recovered the tissue morphology of colon and liver.

\section{BMSCs homing was observed in damaged colon and liver tissues}

In order to confirm the tissue location of therapeutic BMSCs in model mice, BrdU was used to trace stem cells. Our results showed that there were obvious BMSCs homing in inflamed foci of colon (Fig 4a) and liver (Fig 4b) the fifth day after BMSCs treatment. These results prompted that BMSCs might inhibit inflammation and repair damage tissue via directly homing to the inflamed foci.

\section{BMSCs reduced DSS-induced inflammation and ameliorated hepatic function}

We further detected the levels of inflammatory cytokines in liver and serum by ELISA. As can be seen in Fig. 5, levels of serum TNFa, IL-6, IFN $\gamma$ and LPS were elevated markedly after treatment with DSS as compared to the control group, and by contrast, BMSCs could reduce notably DSS-induced serum inflammation (Fig 5a-d). Similarly, elevated liver TNFa and IFN $\gamma$ induced by DSS were suppressed BMSCs transplantation (Fig 5e-f). Meanwhile, serum biochemical analyses were performed to detect hepatic function, we found that the serum ALT, AST, ALP and TBIL levels were extremely increased in the DSS group, and compared with DSS group, BMSCs could markedly reduce these indexes in serum (Fig 5g-j). As HGF play important roles in colon and liver tissue regeneration [35-36], HGF 、 HGF activator (HGFA) and HGF $\alpha$ chain were detected in liver tissues, the results revealed that loss of HGF and HGFA induced by DSS in liver were remarkably reversed by BMSCs (Fig 5k-n). 


\section{BMSCs improved DSS-induced intestinal-liver barrier dysfunction}

To determine the epithelial barrier dysfunction, we detected the expression of tight junction proteins in colon and liver tissues. Our results revealed that tight junction-associated protein Claudin2、Claudin5、 Claudin7、 Occludin and ZO-1 seriously reduced in colon tissue of colitis mouse, indicating DSS induced intestinal barrier dysfunction. After treatment with BMSCs, Claudin2 and Claudin5 were significantly recovered, which indicated that BMSCs partly improved DSS-induced intestinal barrier dysfunction (Fig 6). As the same, the expression of Claudin1、Claudin5、Claudin7、Occludin and ZO-1 were decreased in liver tissue of colitis mouse, while BMSCs remarkably up-regulated these above junction-associated proteins (Fig 6).

\section{Discussion}

In the recent years, the number of patients with IBD accompanied with liver injury continues to increase [37]. It is urgent to clarify the pathogenesis of ulcerative colitis induced liver injury and find appropriate drug interventions [38]. In this current study, we demonstrated that BMSCs alleviated ulcerative colitis induced liver injury via repairing the intestinal-liver barrier and activating hepatocyte growth factor, it has potential application prospects in the treatment of liver injury induced by ulcerative colitis.

Previous studies revealed that owing to the loss of tight junction proteins in the intestinal epithelium, there was extreme increase of intestinal vascular permeability in ulcerative colitis, which would lead to enterotoxins exposure and inflammation induced liver injury, and meanwhile accompanied by tight junction damage in liver tissue and changes in tight junction protein expression [39-41]. Current researches indicate that colon-liver tight junction complex is composed of membrane protein occludin claudins and intracellular protein ZO-1 and other components, of which claudin proteins are the functional and structural basis of tight junction complex. claudin1, claudin2 and claudin3 proteins are found in mature hepatocytes and distributed along bile ducts. Claudins can directly affect the transmembrane electrical impedance of epithelial and endothelial tissues, and regulate the permeability of tight junction. Lack of claudins will cause the damage of endothelial barrier [42-44]. ZO-1 protein plays an important role in assembling mature tight junction structure and maintaining the integrity of tight junction [45-46]. In this study, we demonstrated that BMSCs directly homing to the sites of inflammation both in colon and liver, and repaired the the intestinal-liver barrier via increasing the expression of tight junction proteins, our research provided direct evidences for BMSC localization and repair the tight 
junction and maintaining the integrity of the intestinal-liver barrier.

Hepatocyte growth factor (HGF) is a multifunctional growth factor that plays an important role in the process of development, tissue regeneration and repair. Studies revealed that in damaged colon or liver tissues, HGF was activated by HGFA (hepatocyte growth factor activator), and exert biological activity in tissue regeneration as a form of HGF alpha chain [35-36]. However, whether BMSCs promote activation of HGF is still unknown. Our results revealed that BMSCs significantly increased the HGFA and HGF alpha chain in liver, which indicated that HGF was involved in therapeutic process of BMSCs in liver tissue.

In summary, this study delineated that BMSCs treatment protected DSS-induced liver injury by remodeling the intestinal-liver barrier and inhibiting inflammation response. These results suggested that BMSCs could be used as a promising adjuvant therapeutic strategy in the treatment of colitis-induced liver injury.

\section{Abbreviations}

UC: Ulcerative colitis; BMSCs: bone marrow-derived mesenchymal stem cells; DSS: dextran sodium sulphate; IBD: inflammatory bowel disease; GVHD: graft vs. host disease; HGF: hepatocyte growth factor; AST: aminotransferase; ALT: alanine aminotransferase; ALP: alkaline phosphatase; TBIL: total bilirubin; ELISA: Enzyme-linked immunosorbent assay; DMEM: Dulbecco's modified Eagle's medium; H\&E: hematoxylin and eosin.

\section{Declarations}

\section{Ethics approval and consent to participate}

The experimental protocol was reviewed and approved by the Animal Ethics Committee of Guilin Medical University (No. 20140304026).

\section{Consent for publication}

Not applicable.

\section{Availability of data and materials}

All data generated or analyzed during this study are included in this article.

\section{Competing interests}

The authors declare that they have no competing interests.

\section{Funding}

This study was supported by National Natural Science Foundation of China (no. 81560094) and Guangxi Natural Science Foundation (no.2015GXNSFAA139222).

\section{Authors' contributions}

ZJD and QFZ designed the research and performed the experiments. YCM, LZ and WJL prepared the 
figures, analyzed the data, and drafted the manuscript. JZ and $\mathrm{JH}$ designed the research, analyzed the data. HXJ designed the study, discussed the data, and wrote and reviewed the manuscript. All authors have read and approved the final version of the manuscript for publication.

\section{Acknowledgements}

Not applicable.

\section{Authors' information}

${ }^{1}$ Guangxi Medical University, Nanning 530021, China.

${ }^{2}$ Nanxishan Hospital of Guangxi Zhuang Autonomous Region, Guilin 541002, China.

${ }^{3}$ Guilin Medical University, Guilin 541002, China.

\section{Reference}

1. Karsten Conrad, Dirk Roggenbuck, Martin W Laass. Diagnosis and classification of ulcerative colitis. Autoimmun Rev. 2014;13(4-5):463-6.

2. Lloyd-Price J, Arze C, Ananthakrishnan AN, et al. Multi-omics of the gut microbial ecosystem in inflammatory bowel diseases. Nature. 2019;569(7758):655-662.

3. Halling ML, Kjeldsen J, Knudsen T, et al. Patients with inflammatory bowel disease have increased risk of autoimmune and inflammatory diseases. World J Gastroenterol. 2017;23(33):6137-6146.

4. Uhlig HH, Schwerd T, Koletzko S, et al. The diagnostic approach to monogenic very early onset inflammatory bowel disease. Gastroenterology. 2014;147(5):990-1007.e3.

5. Frank DN, St Amand AL, Feldman RA, et al. Molecular-phylogenetic characterization of microbial community imbalances in human inflammatory bowel diseases. Proc Natl Acad Sci U S A. 2007;104(34):13780-5.

6. Fischer M, Kao D, Kelly C, et al. Fecal Microbiota Transplantation is Safe and Efficacious for Recurrent or Refractory Clostridium difficile Infection in Patients with Inflammatory Bowel Disease. Inflamm Bowel Dis. 2016;22(10):2402-9.

7. Bron PA, Kleerebezem M, Brummer RJ, et al. Can probiotics modulate human disease by impacting intestinal barrier function? Br J Nutr. 2017;117(1):93-107.

8. Martini E, Krug SM, Siegmund B, et al. Mend Your Fences: The Epithelial Barrier and its Relationship With Mucosal Immunity in Inflammatory Bowel Disease. Cell Mol Gastroenterol Hepatol. 2017;23;4(1):33-46.

9. Nakamoto N, Sasaki N, Aoki R, et al. Gut pathobionts underlie intestinal barrier dysfunction and liver T helper 17 cell immune response in primary sclerosing cholangitis. Nat Microbiol. 2019;4(3):492-503.

10. Huang S, Fu Y, Xu B, et al. Wogonoside alleviates colitis by improving intestinal epithelial barrier function via the MLCK/pMLC2 pathway. Phytomedicine. 2020;68:153179.

11. Oriishi T, Sata M, Toyonaga A, et al. Evaluation of intestinal permeability in patients with inflammatory bowel disease using lactulose and measuring antibodies to lipid A. Gut. 1995;36(6):891-6.

12. Targan SR, Karp LC. Defects in mucosal immunity leading to ulcerative colitis. Immunol Rev. 2005;206:296-305.

13. Lidar $M$, Langevitz $P$, Shoenfeld $Y$. The role of infection in inflammatory bowel disease: initiation, exacerbation and protection. Isr Med Assoc J. 2009;11(9):558-63. 
14. Ding L, Yang L, Wang Z, et al. Bile acid nuclear receptor FXR and digestive system diseases. Acta Pharm Sin B. 2015;5(2):135-44.

15. Burge K, Gunasekaran A, Eckert J, et al. Curcumin and Intestinal Inflammatory Diseases: Molecular Mechanisms of Protection. Int J Mol Sci. 2019;20(8):1912.

16. Ronaghan NJ, Shang J, lablokov V, et al. The serine protease-mediated increase in intestinal epithelial barrier function is dependent on occludin and requires an intact tight junction. Am J Physiol Gastrointest Liver Physiol. 2016;311(3):G466-79.

17. Nakamoto N, Sasaki N, Aoki R, et al. Gut pathobionts underlie intestinal barrier dysfunction and liver Thelper 17 cell immune response in primary sclerosing cholangitis. Nat Microbiol. 2019;4(3):492-503.

18. Nakajima H, Munakata A, Yoshida Y. Adverse effects of sulfasalazine and treatment of ulcerative colitis with mesalazine. J Gastroenterol. 1995;30 Suppl 8:115-7.

19. Naganuma M, Sugimoto $S$, Suzuki $H$, et al. Adverse events in patients with ulcerative colitis treated with indigo naturalis: a Japanese nationwide survey. J Gastroenterol. 2019;54(10):891-896.

20. Naganuma M, Sugimoto S, Mitsuyama K, et al. Efficacy of Indigo Naturalis in a Multicenter Randomized Controlled Trial of Patients With Ulcerative Colitis. Gastroenterology. 2018;154(4):935-947.

21. Kastrinaki MC, Pavlaki K, Batsali AK, et al. Mesenchymal stem cells in immune-mediated bone marrow failure syndromes. Clin Dev Immunol. 2013;2013:265608.

22. Liao L, Shi B, Chang H, et al. Heparin improves BMSC cell therapy: Anticoagulant treatment by heparin improves the safety and therapeutic effect of bone marrow-derived mesenchymal stem cell cytotherapy. Theranostics. 2017;7(1):106-116.

23. Li X, Zhan J, Hou Y, et al. Coenzyme Q10 Regulation of Apoptosis and Oxidative Stress in $\mathrm{H}(2) \mathrm{O}(2)$ Induced BMSC Death by Modulating the Nrf-2/NQO-1 Signaling Pathway and Its Application in a Model of Spinal Cord Injury. Oxid Med Cell Longev. 2019;2019:6493081.

24. Zhang J, Brodie C, Li Y, et al. Bone marrow stromal cell therapy reduces proNGF and p75 expression in mice with experimental autoimmune encephalomyelitis. J Neurol Sci. 2009;279(1-2):30-8.

25. Li Z, Liu F, He X, et al. Exosomes derived from mesenchymal stem cells attenuate inflammation and demyelination of the central nervous system in EAE rats by regulating the polarization of microglia. Int Immunopharmacol. 2019;67:268-280.

26. Yin F, Battiwalla M, Ito S, et al. Bone marrow mesenchymal stromal cells to treat tissue damage in allogeneic stem cell transplant recipients: correlation of biological markers with clinical responses. Stem Cells. 2014;32(5):1278-88.

27. Liao L, Yu Y, Shao B, et al. Redundant let-7a suppresses the immunomodulatory properties of BMSCs by inhibiting the Fas/FasL system in osteoporosis. FASEB J. 2018;32(4):1982-1992.

28. $\mathrm{Bi} \mathrm{H}, \mathrm{He} \mathrm{J}, \mathrm{He} \mathrm{X}$, et al. Bone marrow stem cells therapy alleviates vascular injury in a chronic obstructive pulmonary disease-obstructive sleep apnea overlap syndrome rat model. Mol Med Rep. 2021;23(1):69.

29. Dadheech N, Srivastava A, Vakani M, et al. Direct lineage tracing reveals Activin-a potential for improved pancreatic homing of bone marrow mesenchymal stem cells and efficient $ß$-cell regeneration in vivo. Stem Cell Res Ther. 2020;11(1):327.

30. Shichinohe H, Houkin K. Cell Therapy for Stroke: Review of Previous Clinical Trials and Introduction of Our New Trials. Neurol Med Chir (Tokyo). 2016;56(10):592-596. 
31. He Y, Chen D, Yang L, et al. The therapeutic potential of bone marrow mesenchymal stem cells in premature ovarian failure. Stem Cell Res Ther. 2018;9(1):263.

32. Zhang R, Ma J, Han J, et al. Mesenchymal stem cell related therapies for cartilage lesions and osteoarthritis. Am J Transl Res. 2019;11(10):6275-6289.

33. Chen Q, Yan L, Wang CZ, et al. Mesenchymal stem cells alleviate TNBS-induced colitis by modulating inflammatory and autoimmune responses. World J Gastroenterol. 2013;19(29):4702-17.

34. Soleimani M, Nadri S. A protocol for isolation and culture of mesenchymal stem cells from mouse bone marrow. Nat Protoc. 2009;4:102-106.

35. Yamamoto $C$, Yagi S, Hori T, et al. Significance of portal venous VEGF during liver regeneration after hepatectomy. J Surg Res. 2010;159(2):e37-43.

36. Ganesan R, Kolumam GA, Lin SJ, et al. Proteolytic activation of pro-macrophage-stimulating protein by hepsin. Mol Cancer Res. 2011;9(9):1175-86.

37. Chen Y, Lu Y, Pei C, et al. Monotropein alleviates secondary liver injury in chronic colitis by regulating TLR4/NF-kappaB signaling and NLRP3 inflammasome. Eur J Pharmacol. 2020;883:173358.

38. Worland $\mathrm{T}$, Chin $\mathrm{KL}$, van Langenberg $\mathrm{D}$, et al. Retrospective study of idiosyncratic drug-induced liver injury from infliximab in an inflammatory bowel disease cohort: the IDLE study. Ann Gastroenterol. 2020 Mar-Apr;33(2):162-169.

39. Zhang C, Chen $\mathrm{H}, \mathrm{He}$ Q, et al. Fibrinogen/AKT/Microfilament Axis Promotes Colitis by Enhancing Vascular Permeability. Cell Mol Gastroenterol Hepatol. 2020;S2352-345X(20)30170-3.

40. Landy J, Ronde E, English N, et al. Tight junctions in inflammatory bowel diseases and inflammatory bowel disease associated colorectal cancer. World J Gastroenterol. 2016;22(11):3117-26.

41. Tan $Y$, Guan $Y$, Sun $Y$, et al. Correlation of intestinal mucosal healing and tight junction protein expression in ulcerative colitis patients. Am J Med Sci. 2019;357(3):195-204.

42. Günzel D, Fromm M. Claudins and other tight junction proteins. Compr Physiol. 2012;2(3):1819-52.

43. Günzel D, Yu AS. Claudins and the modulation of tight junction permeability. Physiol Rev. 2013;93(2):525-69.

44. Garcia-Hernandez V, Quiros M, Nusrat A. Intestinal epithelial claudins: expression and regulation in homeostasis and inflammation. Ann N Y Acad Sci. 2017;1397(1):66-79.

45. Umeda K, Ikenouchi J, Katahira-Tayama S, et al. ZO-1 and ZO-2 independently determine where claudins are polymerized in tight-junction strand formation. Cell. 2006;126(4):741-54.

46. Schwayer C, Shamipour S, Pranjic-Ferscha K, et al. Mechanosensation of tight junctions depends on ZO-1 phase separation and flow. Cell. 2019;179(4):937-952.e18.

\section{Figure legend}

Figure 1. Typical pathological changes in colitis induced liver injury model mouse. (a), Body weight. (b), Colon length. (c), Colonic pathology images. (d), the serum ALT, AST, TC levels. Values presented are expressed as the mean \pm SEM, ${ }^{* *} P<0.001$ versus control group, ${ }^{* *} P<0.01,{ }^{*} P<0.05$ versus the Control group.

Figure 2. The expression of tight junction proteins were abnormal in colon and liver tissues. (a), Representative Western blots of tight junction proteins in colon and liver tissues. The induction folds of (b) 
Claudin1, (c) Claudin2, (d) Claudin5, (e) Claudin7, (f) Occludin and (g) ZO-1 are shown in mean \pm SE of five mice for each group after calculating the intensity of the treatment relative to Control and normalized by $\alpha$-tubulin intensity, ${ }^{* *} P<0.001,{ }^{* *} P<0.01,{ }^{*} P<0.05$ versus the Control group. ns, no statistical significance.

Figure 3. Effect of BMSCs on the clinical symptoms of colitis. (a), \% body weight change. Data expressed as mean \pm SEM ( $\mathrm{n}=15 /$ group). ${ }^{* * *} P<0.001$ vs Control; ${ }^{\#} P<0.01$ vs Control; ${ }^{\&} P<0.05$, ${ }^{\& \&} P<0.01$ vs Model+PBS; (b), colon length, Data expressed as mean \pm SEM ( $n=15 /$ group), ${ }^{* *} P<0.01,{ }^{* * *} P<$ 0.001. (c), Colonic pathology images. (d), Liver pathology images.

Figure 4. BrdU immunofluorescence. Nine days after intravenous transplanted of BMSCs, cells derived from BMSCs were identified by rat anti-BrdU antibody and Alexa 488-conjugated secondary antibody in colon tissue (a) and liver tissue (b). Arrows showed BrdU positive cells derived from endogenous BMSCs.

Figure 5. BMSCs suppressed DSS-induced inflammation and ameliorated colitis induced hepatic dysfunction. Serum inflammatory factors TNF $\alpha$ 、IL6、IFN $\gamma$ and serum LPS were detected (a-d), and TNF $\alpha$, IFN $\gamma$ (e-f). Serum ALT, AST, ALP and TBIL levels were detected to assess hepatic function (g-j). HGF and HGFA were evaluated in liver tissue by ELISA (k-I). Meanwhile, HGF $\alpha$ chain were detected by western blot in in liver tissue $(m-n)$. Data expressed as mean \pm SEM $\left(n=15 /\right.$ group). ${ }^{*} P<0.05,{ }^{* *} P<0.01$, ${ }^{* * *} P<0.001$ vs Control; ${ }^{\&} P<0.05$, ${ }^{\& \&} P<0.01$, ${ }^{\& \& \&} P<0.001$ vs Model+PBS. ns, no statistical significance.

Figure 6. Effects of BMSCs in DSS-induced intestinal-liver barrier dysfunction. (a), protein levels of Claudin1、Claudin2、Claudin5、Claudin7、Occludin and ZO-1 in colon and liver tissues were analyzed using western blotting. (b-m), densitometry of Claudin1、Claudin2、Claudin5、Claudin7、Occludin and ZO-1 expression. Band densities were normalized to $\alpha$-tubulin. Data are reported as means $\pm S D$ of three independent experiments. ${ }^{*} P<0.05,{ }^{* *} P<0.01,{ }^{* * *} P<0.001$. ns, no statistical significance $(t$-test). 
Figures

a

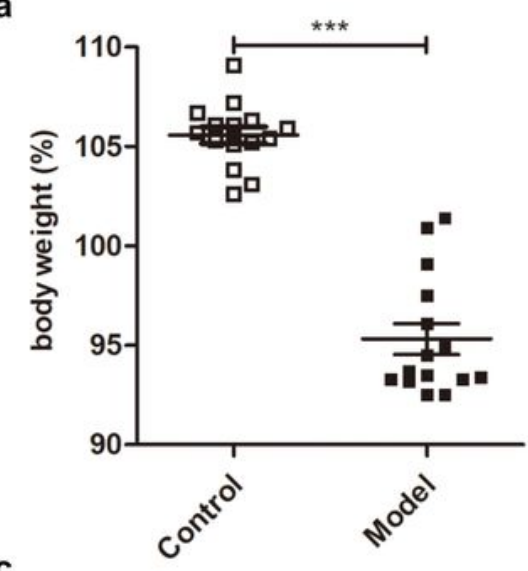

b

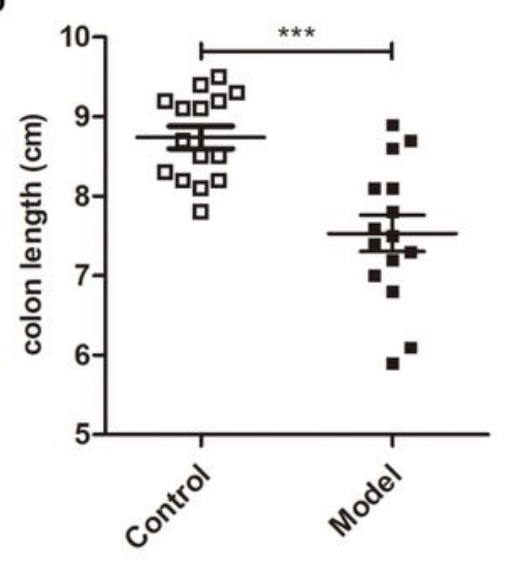

c

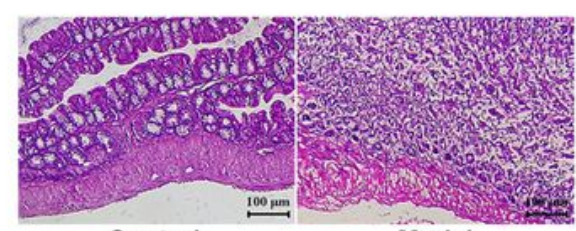

Control Model

d
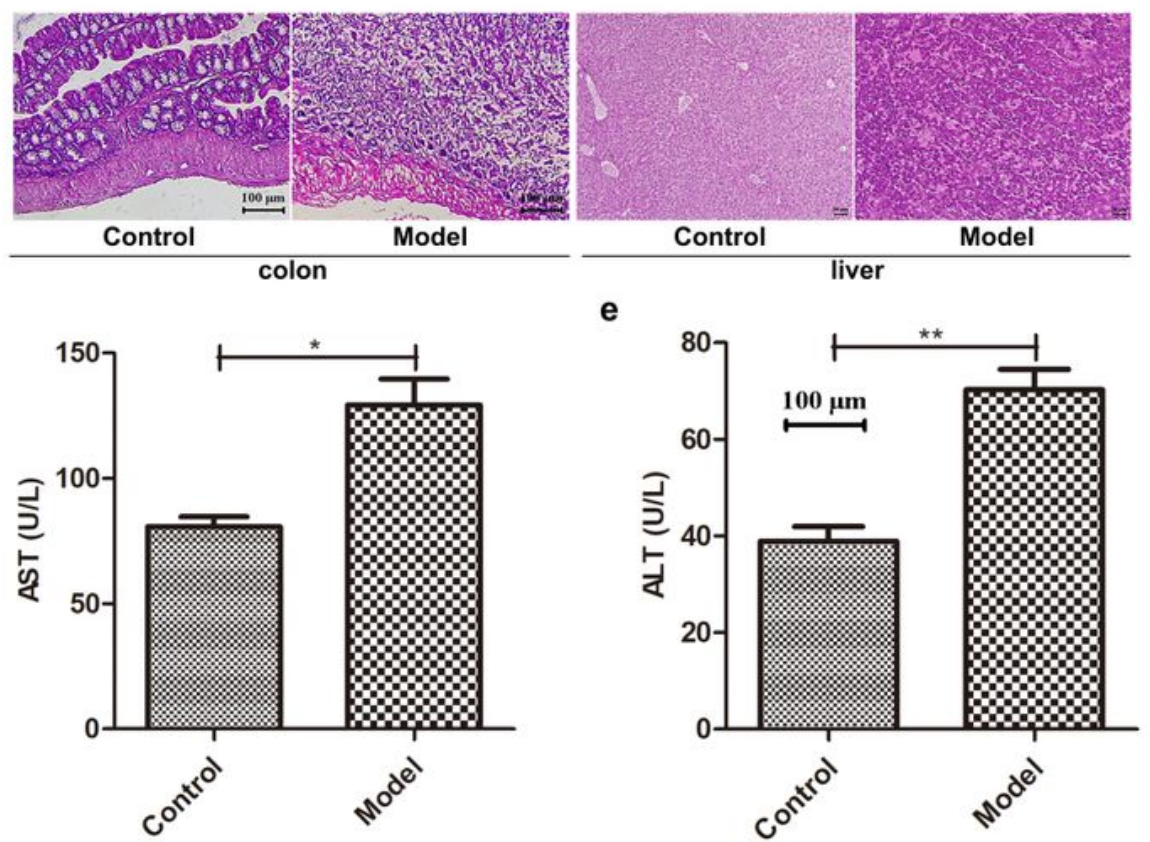

e

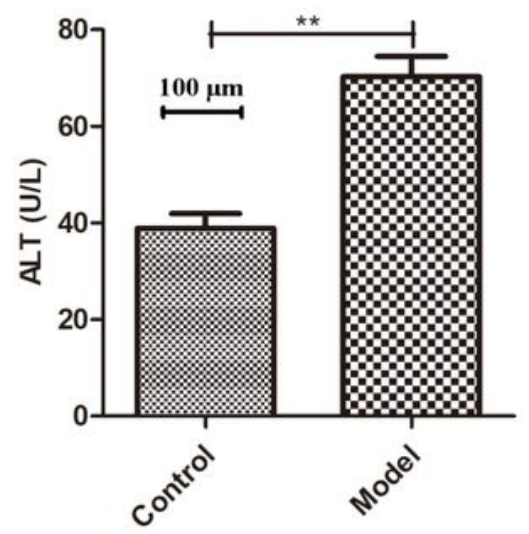

f

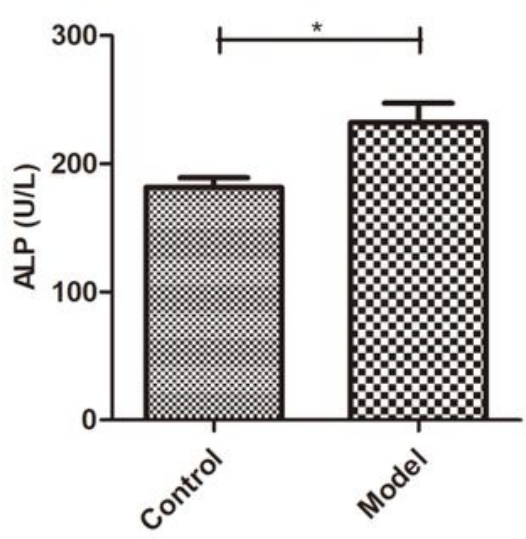

g

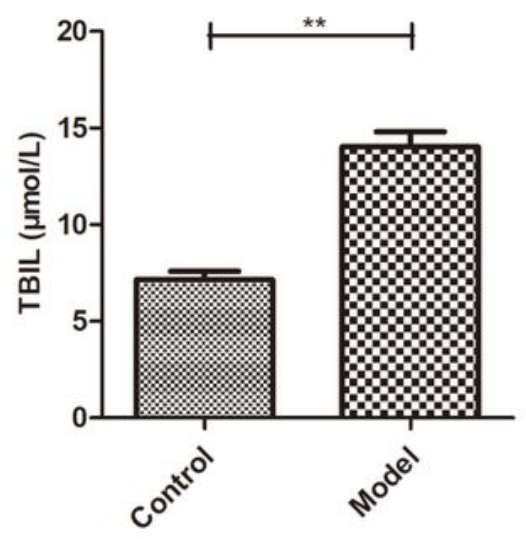

Figure 1

Typical pathological changes in colitis induced liver injury model mouse. (a), Body weight. (b),Colon length. (c), Colonic pathology images. (d), the serum ALT, AST, TC levels. Values presented are expressed

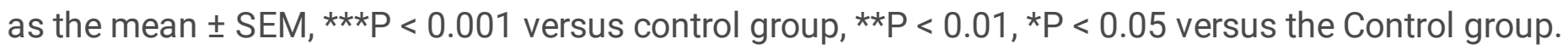


a

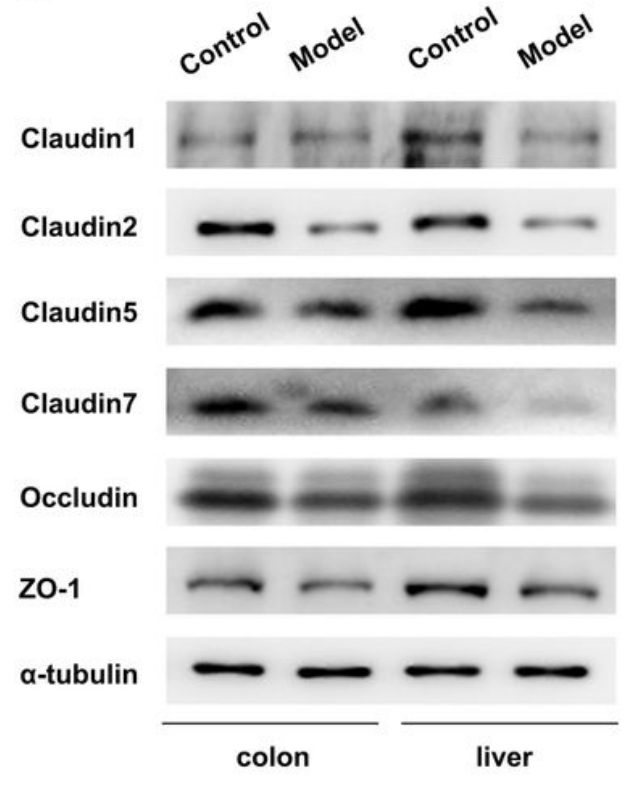

b
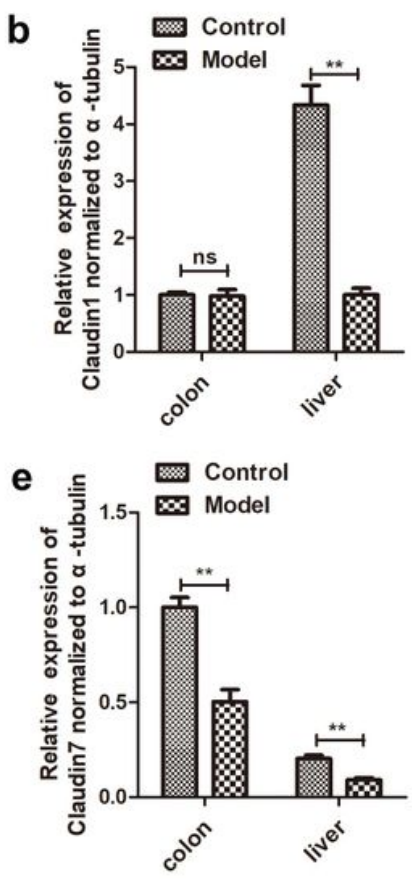
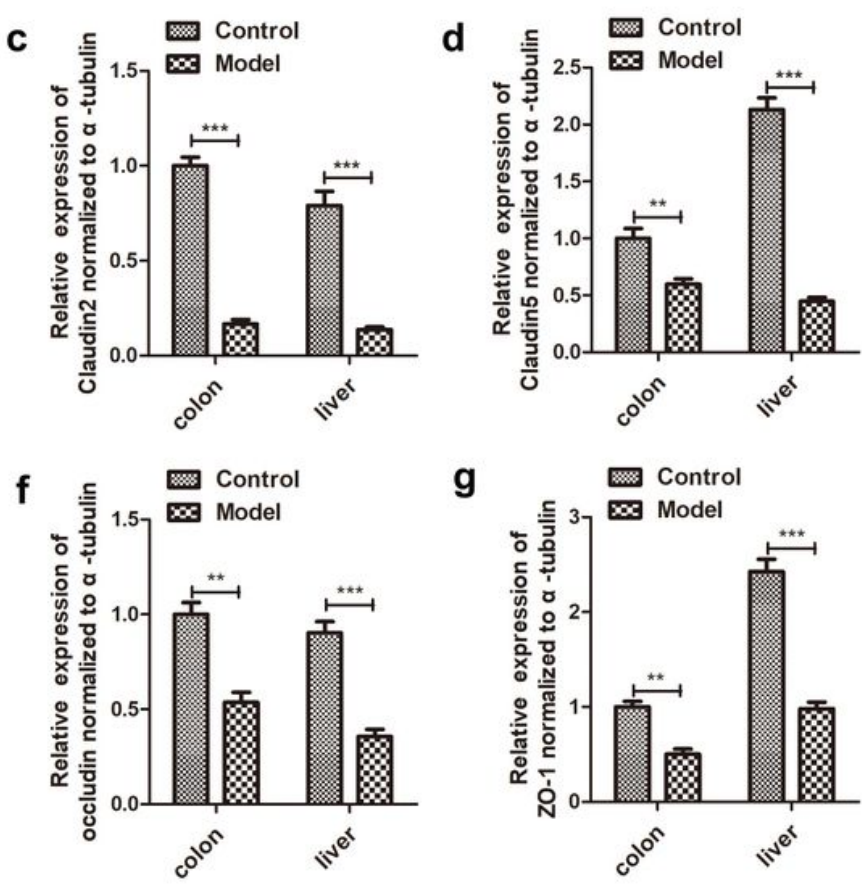

g

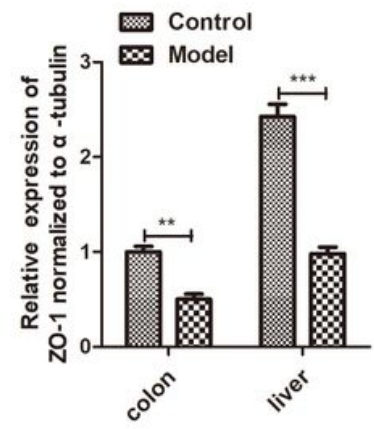

Figure 2

The expression of tight junction proteins were abnormal in colon and liver tissues. (a), Representative Western blots of tight junction proteins in colon and liver tissues. The induction folds of (b) Claudin1, (c) Claudin2, (d) Claudin5, (e) Claudin7, (f) Occludin and (g) ZO-1 are shown in mean \pm SE of five mice for each group after calculating the intensity of the treatment relative to Control and normalized by a -tubulin intensity, ${ }^{\star * \star} \mathrm{P}<0.001,{ }^{\star *} \mathrm{P}<0.01,{ }^{*} \mathrm{P}<0.05$ versus the Control group. ns, no statistical significance. 

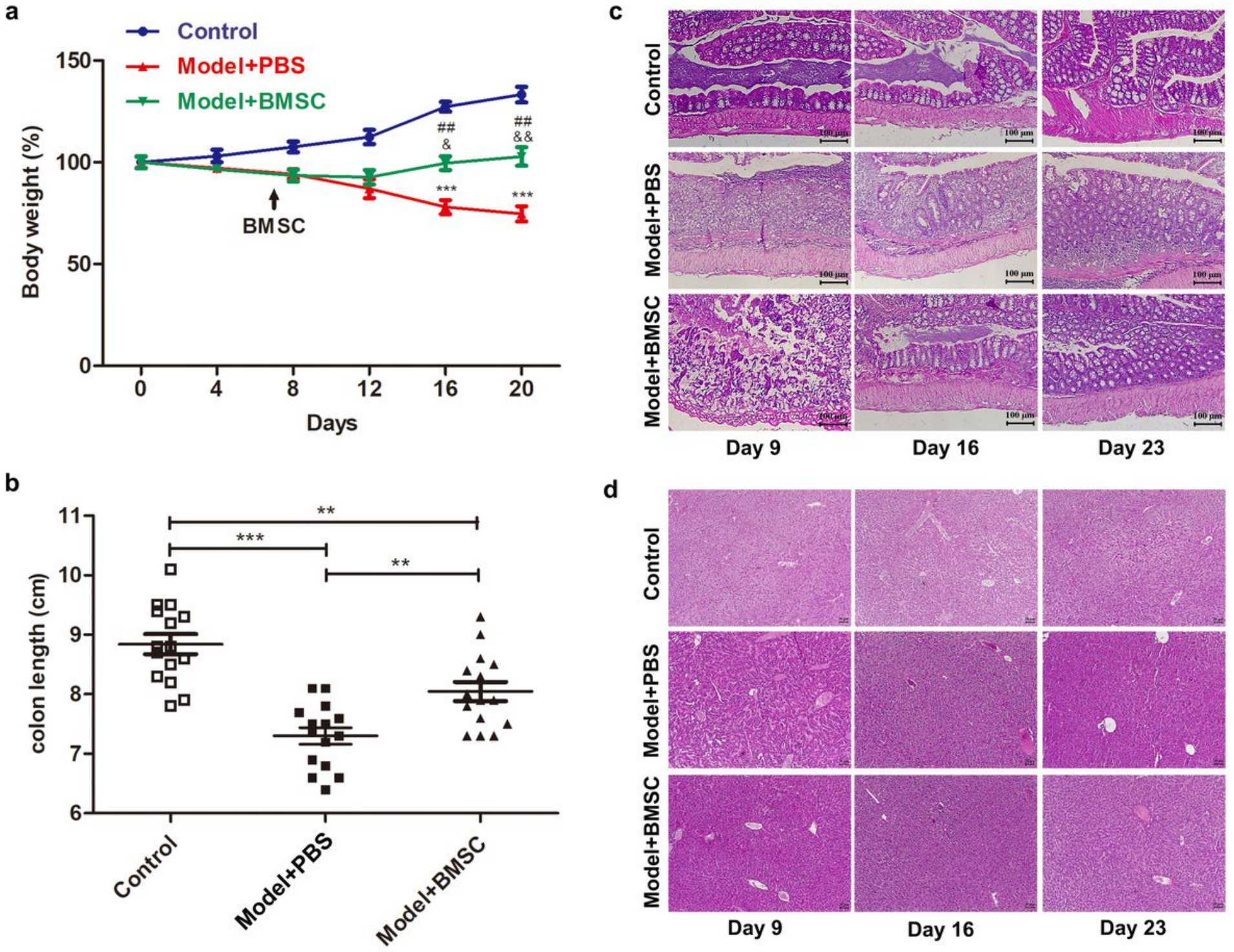

d

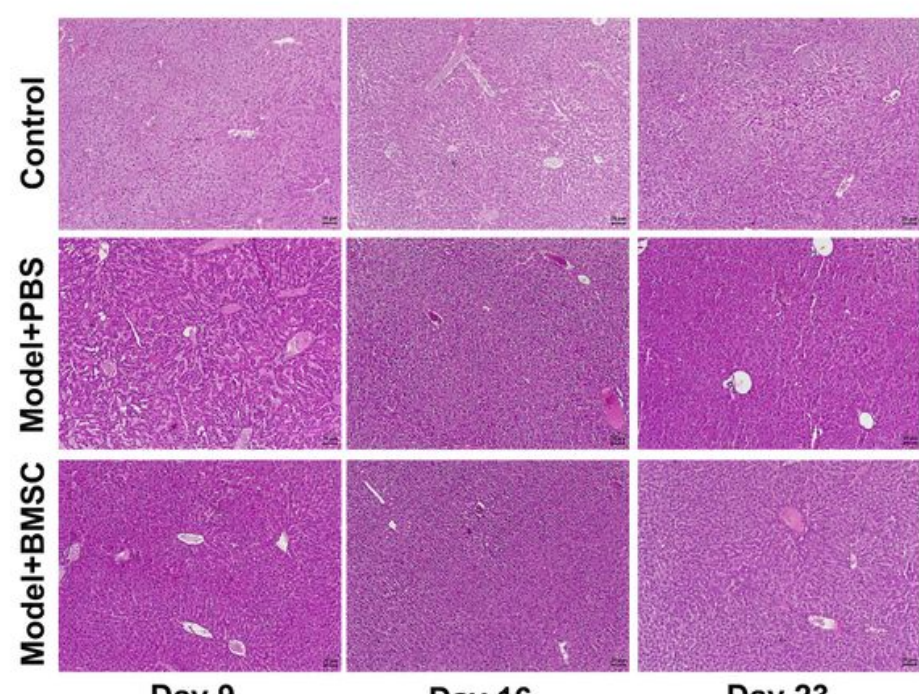

Figure 3

Effect of BMSCs on the clinical symptoms of colitis. (a), \% body weight change. Data expressed as mean

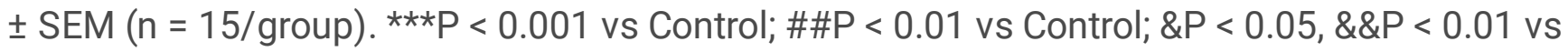
Model+PBS; (b), colon length, Data expressed as mean \pm SEM ( $n=15 /$ group), ${ }^{\star \star} P<0.01,{ }^{\star \star \star *} P<0.001$. (c), Colonic pathology images. (d), Liver pathology images. 

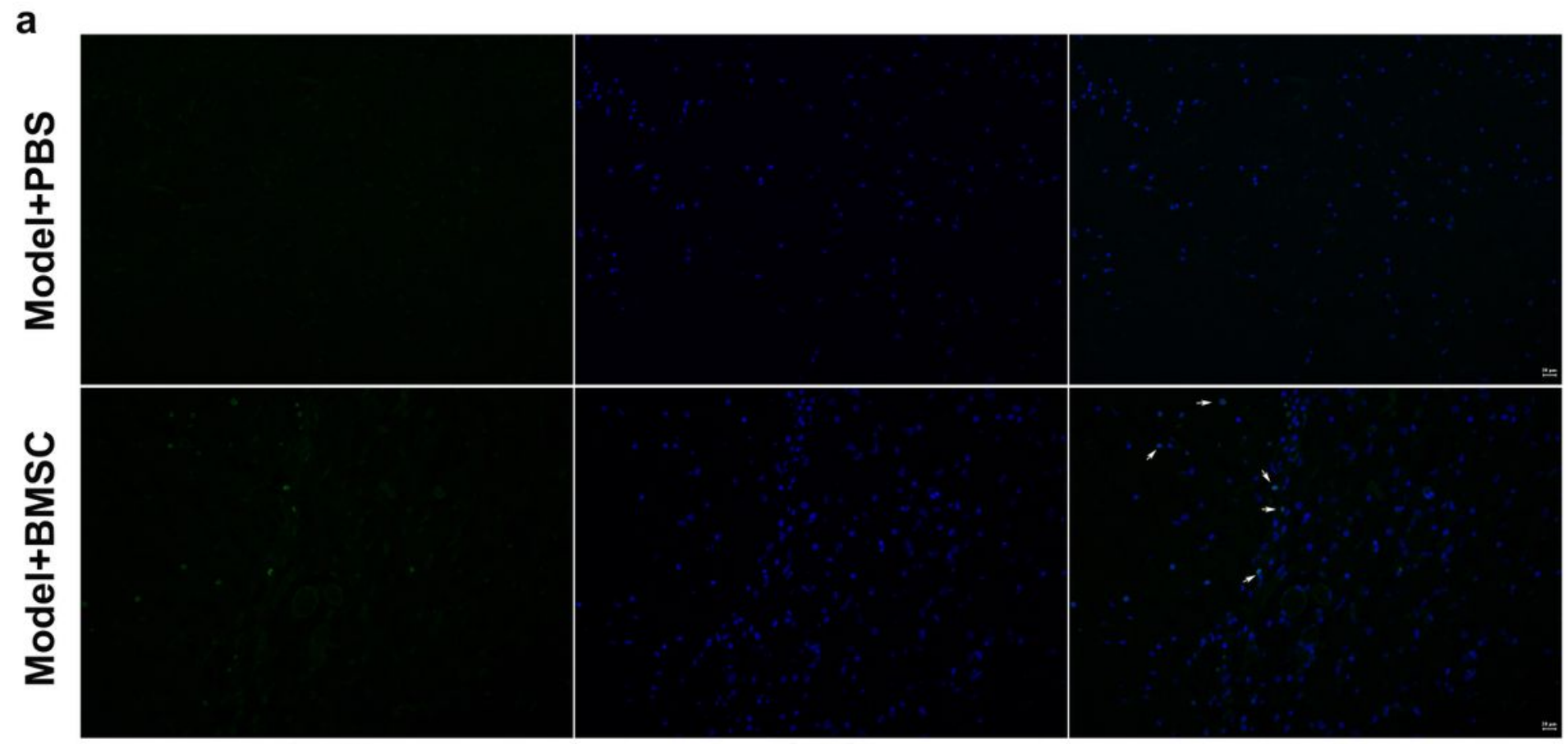

\section{BrdU}

DAPI

Merge

b

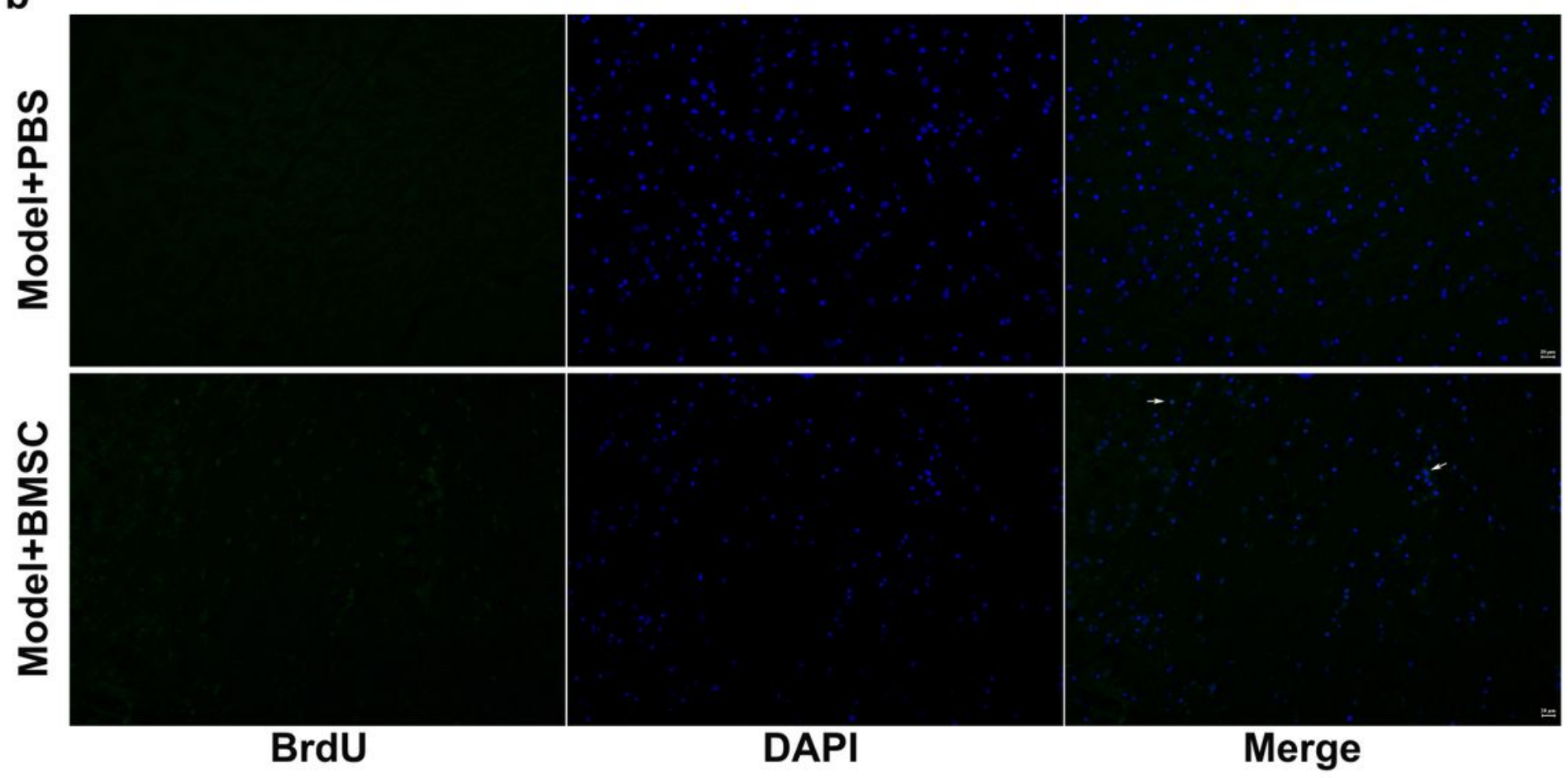

Figure 4

BrdU immunofluorescence. Nine days after intravenous transplanted of BMSCs, cells derived from BMSCs were identified by rat anti-BrdU antibody and Alexa 488-conjugated secondary antibody in colon tissue (a) and liver tissue (b). Arrows showed BrdU positive cells derived from endogenous BMSCs. 
a
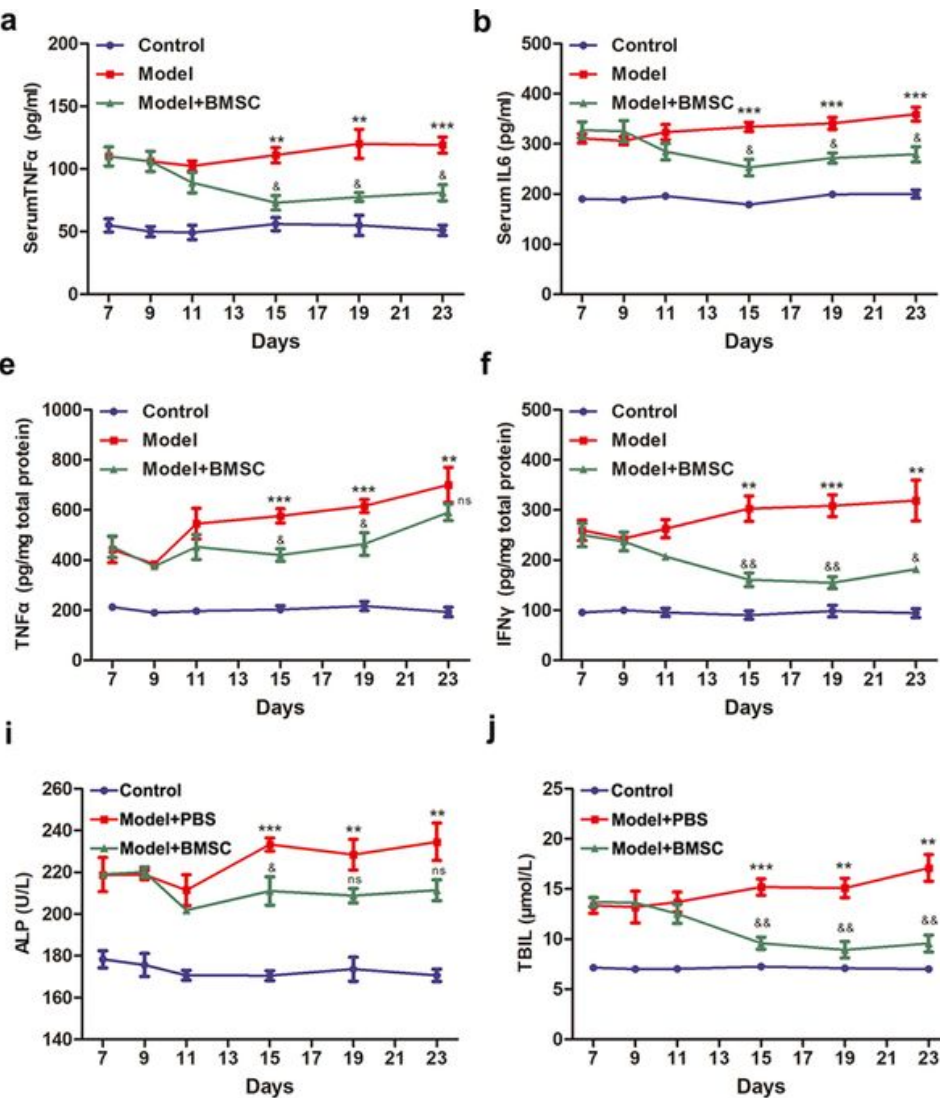

m

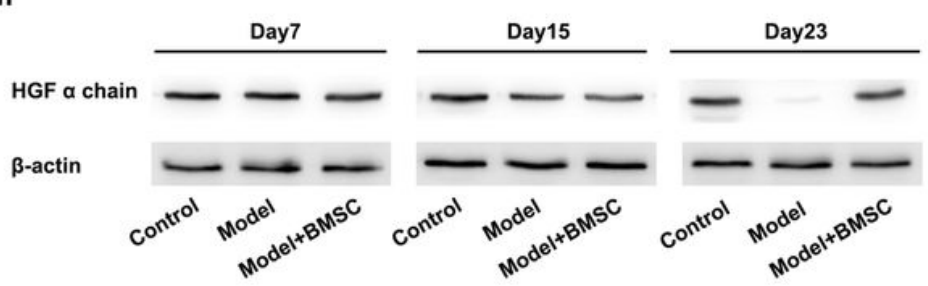

c
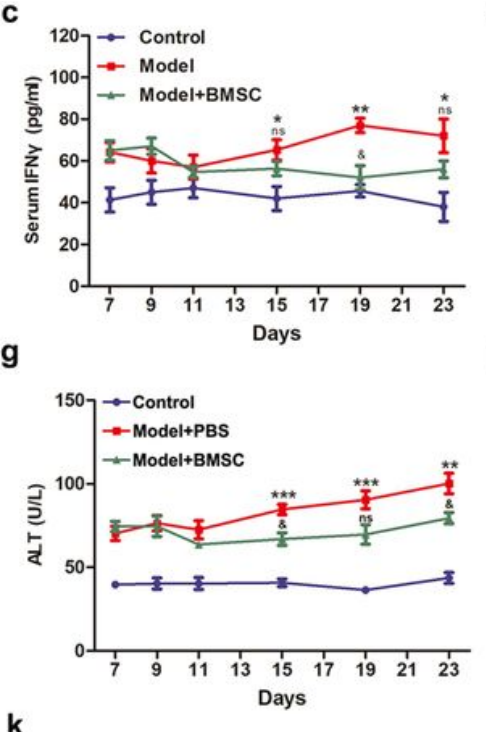

k
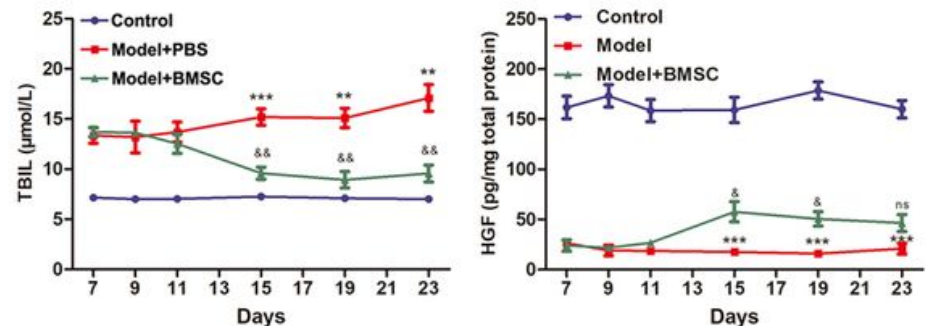

n

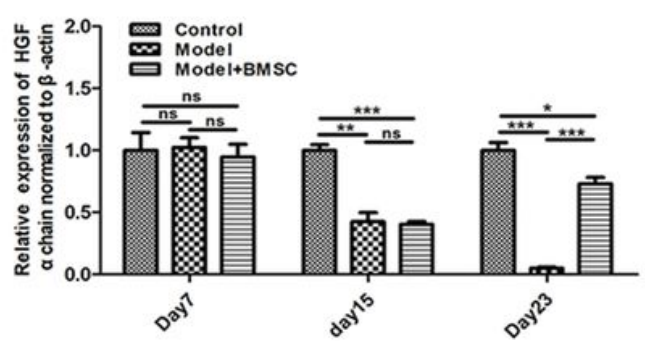

Figure 5

BMSCs suppressed DSS-induced inflammation and ameliorated colitis induced hepatic dysfunction.

Serum inflammatory factors TNFaDIL6IIFNy and serum LPS were detected (a-d), and TNF aDIFNy (e-f).

Serum ALT, AST, ALP and TBIL levels were detected to assess hepatic function (g-j). HGF and HGFA were evaluated in liver tissue by ELISA $(\mathrm{k}-\mathrm{l})$. Meanwhile, HGF a chain were detected by western blot in in liver tissue (m-n). Data expressed as mean \pm SEM ( $n=15$ /group). ${ }^{*} P<0.05$, $* * P<0.01$, ${ }^{\star \star *} P<0.001$ vs Control; \&P $<0.05, \& \& P<0.01, \& \& \& P<0.001$ vs Model+PBS. ns, no statistical significance. 
a

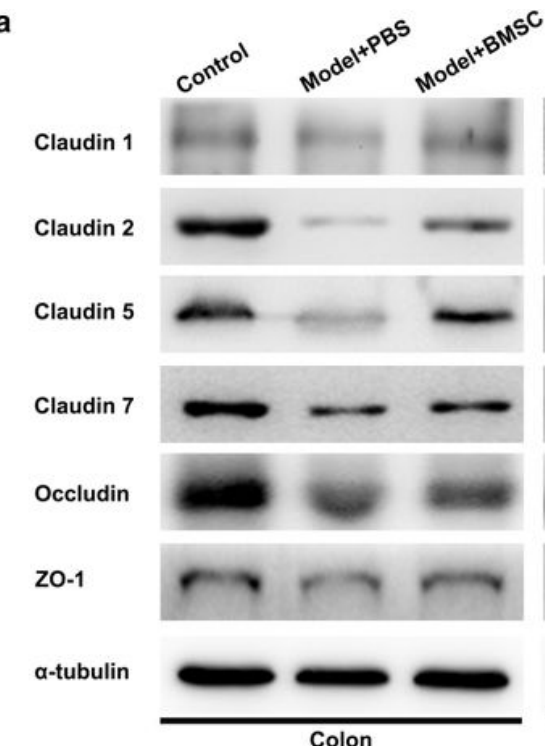

f

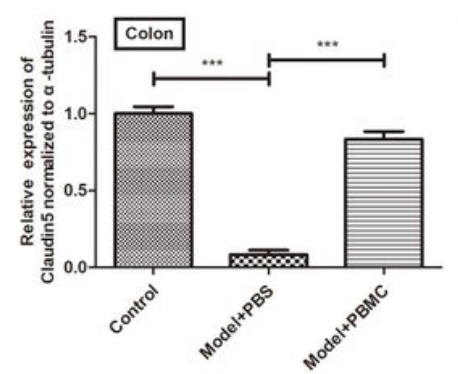

j

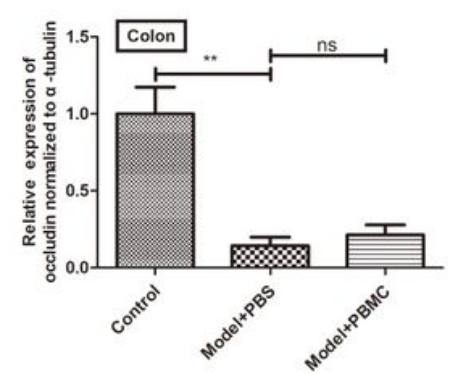

k

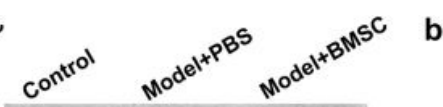

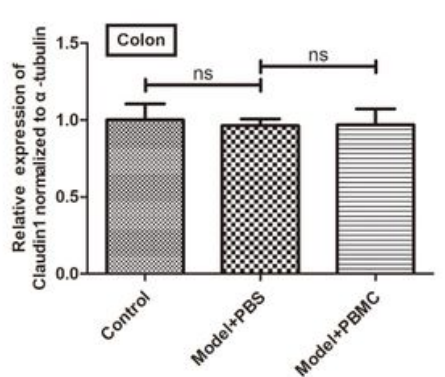

d

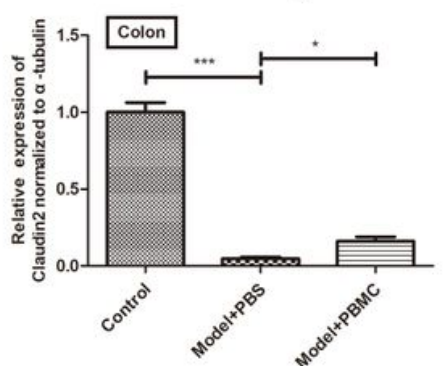

h
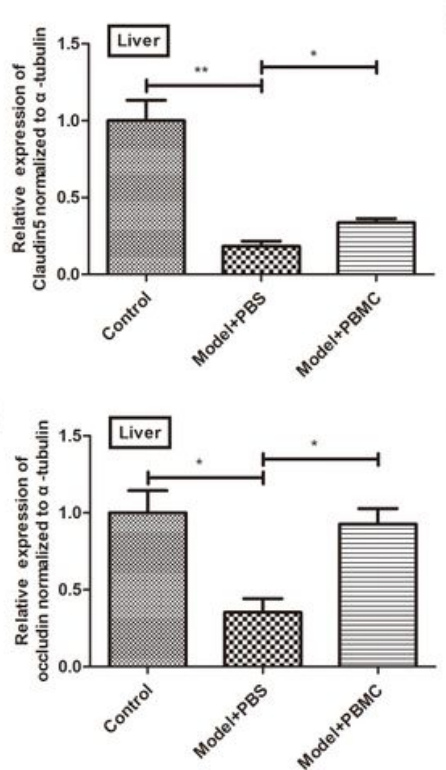

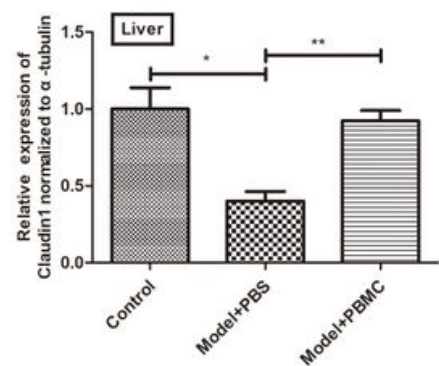

e

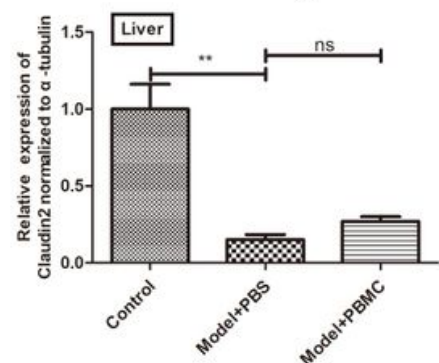

i

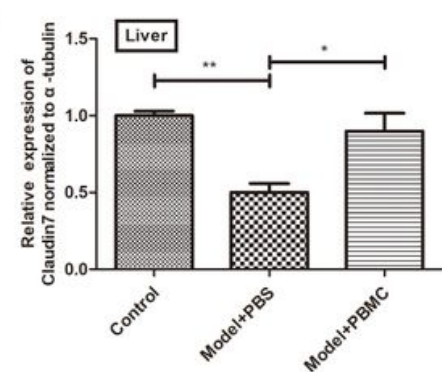

m

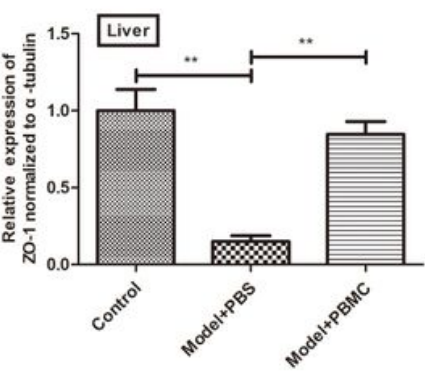

Figure 6

Effects of BMSCs in DSS-induced intestinal-liver barrier dysfunction. (a), protein levels of Claudin10

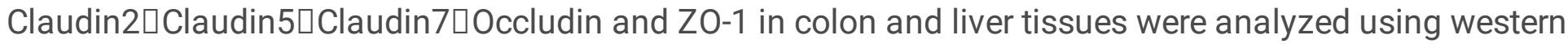

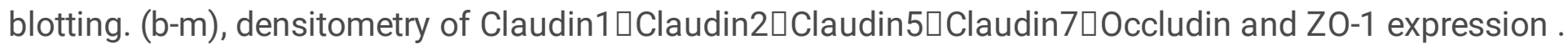
Band densities were normalized to a -tubulin. Data are reported as means $\pm S D$ of three independent experiments. ${ }^{*} P<0.05,{ }^{*} P<0.01,{ }^{*} * *<<0.001$. ns, no statistical significance (t-test). 